\title{
THE SIGNIFICANCE OF THE TRENDELENBURG TEST
}

\author{
PHILIP HARDCASTLE. SYDNEY NADE
}

From the University of Western Australia

\begin{abstract}
Trendelenburg's test of function of the hip joint was first reported before radiology was available. At least four methods of performing it have since been described in the literature.

We examined 50 normal subjects and 103 people with disorders affecting either the spine or the hip, in order to determine the different responses that occurred when they were asked to stand on one leg. This has enabled us to define a standard method of performing the Trendelenburg test, and to interpret the test as a method of assessing hip abductor function.

The major pitfalls that result in misinterpretation, or false-positive responses, are pain, lack of cooperation from the patient, and impingement between the rib cage and the iliac crest. False-negative responses result from the patient using muscles above and below the pelvis, and from leaning beyond the hip on the standing side.
\end{abstract}

In 1897 Friedrich Trendelenburg described a test which he found useful in determining the integrity of hip abductor muscle function, with specific reference to congenital dislocation of the hip and progressive muscular atrophy (Rang 1966). Trendelenburg's report (Fig. 1) appeared only two years after the discovery of $\mathrm{x}$-rays by Roentgen, at a time when the physician had few aids to diagnosis other than his ears, eyes, and fingers. The test is mentioned in most major orthopaedic and physiotherapy textbooks as a diagnostic manoeuvre to be carried out in the assessment of function and dysfunction of the hip.

In the standard textbooks we found at least four different methods of performing the Trendelenburg test, usually described vaguely, and it was seldom clear how the test should be interpreted. Furthermore, there was little agreement among our colleagues about the performance or interpretation of the test, although most of them had routinely carried out their own version of it on many of their patients. Because of this confusion as to the method, meaning and value of the test, we decided to investigate it.

The aims of this study were, first, to record the responses of normal people to the request to stand on one leg, and hence to define a standard Trendelenburg test: and second, to study people with various abnormalities of the hip and its muscles in order to assess the value and the pitfalls of the test as used in current orthopaedic practice.

P. H. Hardcastle. FRACS. Senior Lecturer in Orthopaedic Surgery S. M. L. Nade. MD. FRACS. Professor of Orthopaedic Surgery Queen Elizabeth II Medical Centre. Nedlands. Western Australia 6009. Requests for reprints should be sent to Professor S. M. L. Nade. 1 1985 British Editorial Society of Bone and Joint Surgery $0301620 \times 855150 \$ 2.00$
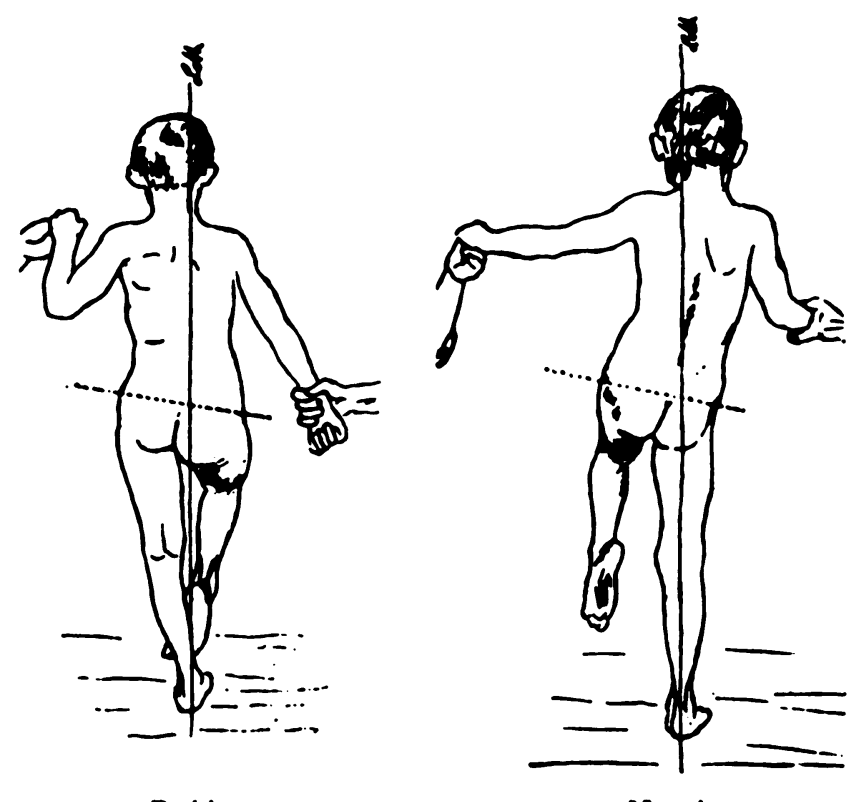

Ponitive

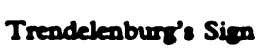

Nequive

Fig. 1

These drawings demonstrate what Trendelenburg described as a positive and negative sign. Note that both hands are held by an assistant and the alignment of the pelvis with respect to the ground is observed. (From Mercer Rang`s Anthology of orthopaedics, with permission).

\section{SUBJECTS}

Normal volunteers. A group of 50 normal people aged between 3 and 50 years were examined to determine what happened to their posture when they stood on one leg. Two positions of the non-stance leg were studied: first the test was performed with the hip flexed to 30 , and then it was repeated with the hip flexed to 90 . Each person was asked to maintain the one-leg stance posture for 30 seconds. 
Volunteers with neuromusculoskeletal disorders. Of the 103 people studied, 12 had bilateral hip disorders. The age range of the 103 patients was from 6 to 82 years. They were divided into two major groups (Table I): those with neurological disorders and those with mechanical disorders of the hip or spine.

\section{DATA ANALYSIS}

Clinical photography and videotape. Initially, colour videotapes were used in order to study the movement patterns by single-frame analysis. As we became more experienced, the responses were recorded on $35 \mathrm{~mm}$ colour projection slides.

Electromyography. This was performed on three normal volunteers. Gluteus maximus, gluteus medius, gluteus minimus, tensor fascia lata and adductor magnus muscle activities were recorded with the subject at rest and subsequently with the pelvis in the different positions found to occur regularly when the test was performed. Three walking subjects with incomplete paraplegia due to traumatic fracture-dislocation were also studied.

Assessment of abductor muscle power. With the subject lying on one side, the strength of abduction of the hip was assessed, using the Medical Research Council grading of muscle power.

\section{RESULTS}

\section{Normal responses}

With the non-stance hip flexed at 30 . Three different patterns of pelvic and spinal movement occurred with the non-stance hip flexed to 30 .

Response 1. The pelvis rose on the non-stance side, the typical description of a "negative Trendelenburg test";
Table I. Classification of the neuromusculoskeletal disorders of the 103 volunteers

1. Neurological conditions

Incomplete paraplegia Muscular dystrophy

Nerve root entrapment

Cerebral palsy

Poliomyelitis

Hemiplegia

2. Mechanical disorders

(a) Of the spine

Scoliosis

Ankylosing spondylitis

Iliac crest defect after

anterior spinal fusion

(b) Of the hip (i) In children

Congenital dislocation Subluxation

Coxa vara

Perthes" disease

Slipped capital femoral epiphysis

(ii) In adults

Fractured neck of femur

After arthroplasty

Osteoarthritis

Avascular necrosis

* Including one with congenital dislocation of the hip (Fig. 13)

there was a compensatory scoliosis convex to the stance side (Fig. 2).

Response 2. The pelvis remained parallel to the ground with minimal spinal compensation (Fig. 3).

Response 3. The pelvis dropped on the non-stance side and the buttock crease moved downwards. This was associated with adduction of the weight-bearing hip and a compensatory scoliosis convex to the non-stance side, as seen in the classical "positive Trendelenburg test". Balance was achieved by moving the torso and centre of gravity directly over the weight-bearing hip (Fig. 4).

With the non-stance hip flexed 90 . Response 1 . In this position the pelvis rose on the non-stance side but not as

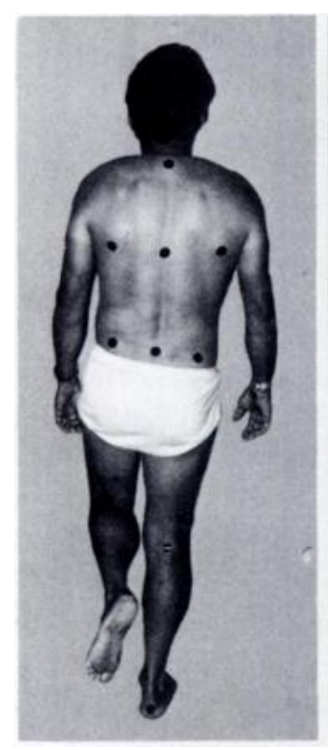

Fig. 2

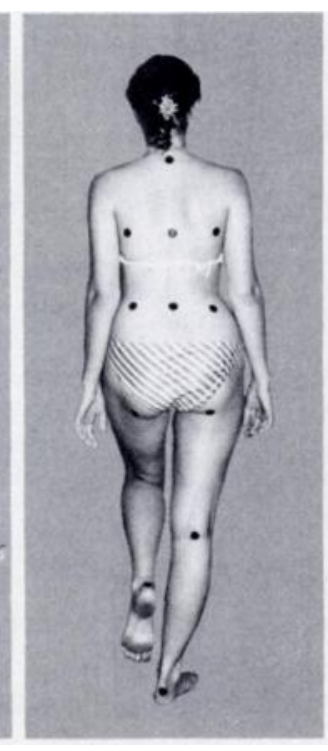

Fig. 3

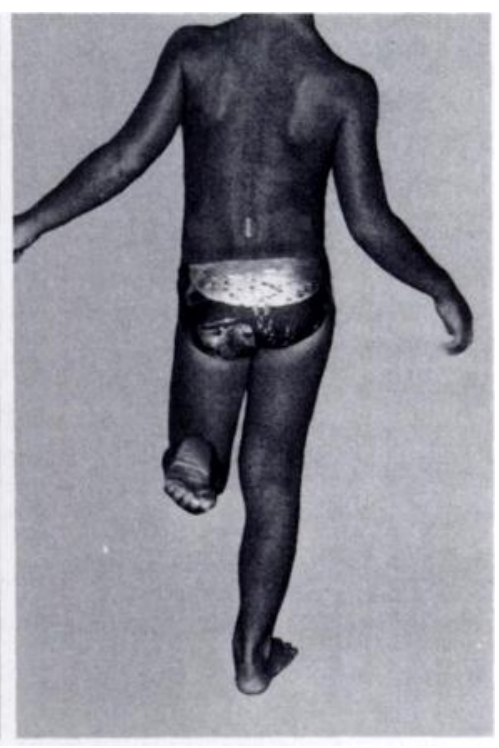

Fig. 4

Three different responses were seen in normal volunteers when they were asked to stand on one leg with the hip on the non-stance side flexed at about 30 . Figure 2 - The pelvis on the nonstance side rose above the stance side with the trunk centred over the stance hip (a negative response). Figure 3-The pelvis remained parallel to the ground. Figure 4-The pelvis on the non-stance side dropped below the level of the stance side (a positive Trendelenburg sign). 
high as when the non-stance hip was flexed only 30 . This was because pelvic rotation brought the iliac crest closer to the rib cage and made spinal compensation uncomfortable.

Response 2. The pelvis remained parallel to the ground.

There was no third response with the hip flexed 90 - in no subject did the pelvis drop on the non-stance side.

Electromyographic findings. Electromyograms were obtained from three volunteers. It was found that with the third response (false-positive test) no activity was present in gluteus maximus, gluteus medius, gluteus minimus or adductor magnus; activity was present in tensor fascia lata. If the subject, on command, voluntarily raised the pelvis on the non-stance side, there was a gradual increase of the hip abductor muscle activity, reaching a maximum when the first response was achieved; no activity was recorded in adductor magnus or gluteus maximus. With the non-stance hip at $90^{\circ}$ abductor activity was the same as during Responses 1 and 2 with the non-stance hip at $30^{\prime \prime}$.

\section{Responses in abnormal subjects}

Recording and classifying the findings in a group of children and adults with a wide spectrum of different conditions proved difficult at first. Because we examined abnormal subjects before we had clarified the variable response patterns in normal people, we had not defined a standard way of performing the test. What became clear was that some people with pathological disorders had a false negative Trendelenburg test (Table II).

Table II. False responses to Trendelenburg test

$\begin{array}{ll}\text { Causes of false negatives } & \text { Causes of false positives } \\ \begin{array}{l}\text { Use of suprapelvic muscles } \\ \text { Use of psoas and rectus femoris }\end{array} & \text { Pain } \\ \begin{array}{l}\text { Wide lateral translocation of trunk } \\ \text { to allow balance over the hip as } \\ \text { a fulcrum }\end{array} & \begin{array}{l}\text { Lack of co-operation or } \\ \text { understanding }\end{array} \\ & \text { Costo-pelvic impingement }\end{array}$

This false negative was particularly evident in patients with neurological disorders, many of whom could raise the pelvis on the non-stance side. They did this, first, by moving the torso well over the weightbearing hip, thereby reducing the amount of abductor activity necessary to maintain this posture (Fig. 5). Secondly, by supporting the hand on a table or wall on the non-stance side, they were then able, by a combination of muscle contraction of the shoulder adductors, especially latissimus dorsi, and (possibly) psoas major on the non-stance side, with quadratus lumborum on the weight-bearing side, to raise the non-stance side of the pelvis above that of the weight-bearing hip (Fig. 6). We also found that people with pain in the hip tended to shift the centre of gravity over the hip to decrease the pull of the abductors. Other patients with a painful hip or spine

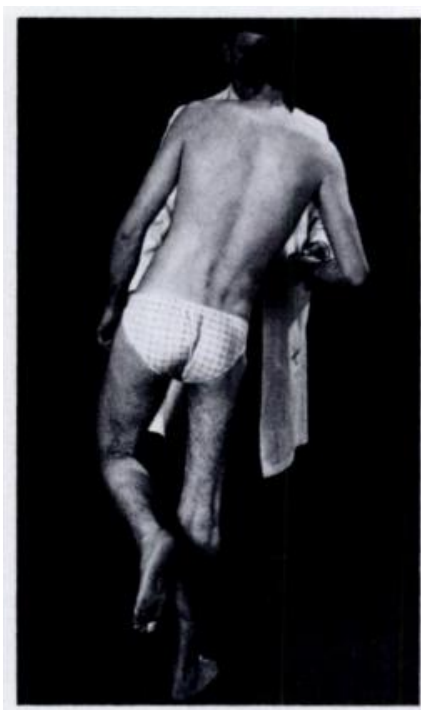

Fig. 5

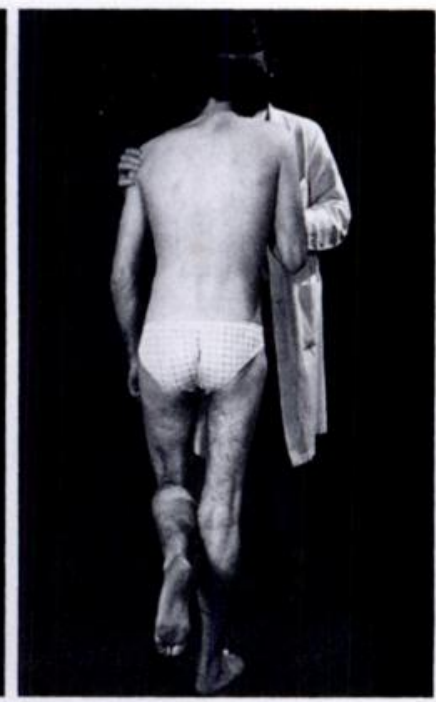

Fig. 6
Figure 5-A false-negative test can occur if the trunk shifts excessively to reduce the abductor muscle force necessary to obtain equilibrium; note the wasting of the right thigh due to poliomyelitis. Figure $6-$ When the trunk is re-aligned over the stance hip the pelvis drops rapidly on the non-stance side because there is not enough muscle power available to maintain the elevated position.

were able to stand on the painful hip for only a very short period. In the absence of significant pain, subjects with weak hip abductor muscles could achieve balance by lifting the pelvis on the non-stance side, using functioning muscles, kinetic energy and gravity.

Electromyography was performed on three patients who could elevate the non-stance side of the pelvis by either leaning well over the hip joint or using muscle groups above the pelvis; minimal electrical activity was recorded in the abductor muscle groups. Thus, simply looking at the final position of the trans-iliac line of the pelvis gives no assessment of abductor muscle function.

It was our experience that children under the age of four years could not be reliably assessed, however the test was performed. In children over four, the Trendelenburg test could be assessed only if the children could understand and co-operate fully; unless this was possible, assessment was invalid, and different responses were seen at separate examinations at short time intervals on the same day.

Delayed positive Trendelenburg sign. Several people had an initial negative test, but after standing for a short time with the non-stance side of the pelvis raised, it gradually began to fall and they were not able to maintain their initial posture. This has been called a delayed positive Trendelenburg sign: Mitchell (1973) suggested that the time at which the pelvis began to drop should be recorded. In people with a delayed positive Trendelenburg sign the gait can be normal, but when they are asked to walk quickly, it becomes apparent that they fatigue easily and a limp, with all the characteristics of the so-called Trendelenburg gait, becomes obvious.

Two subjects with severe scoliosis had false-positive responses because of impingement between the lower 


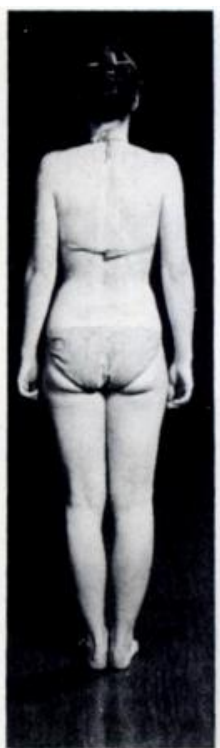

Fig. 7

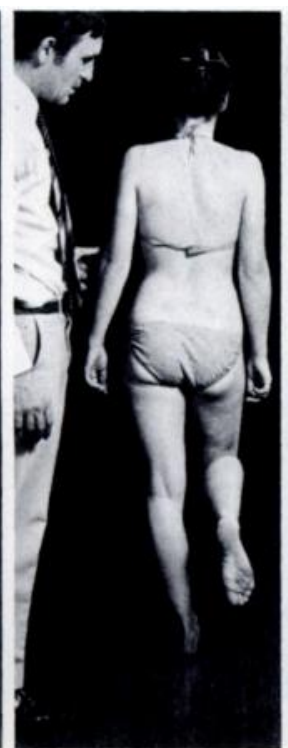

Fig. 8

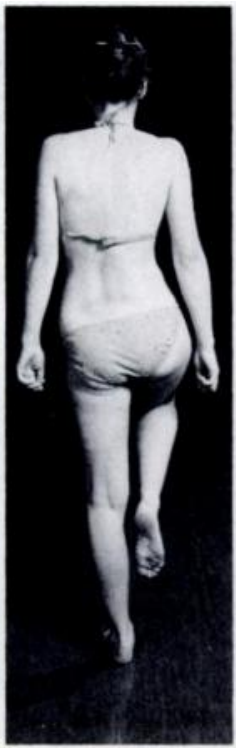

Fig. 9

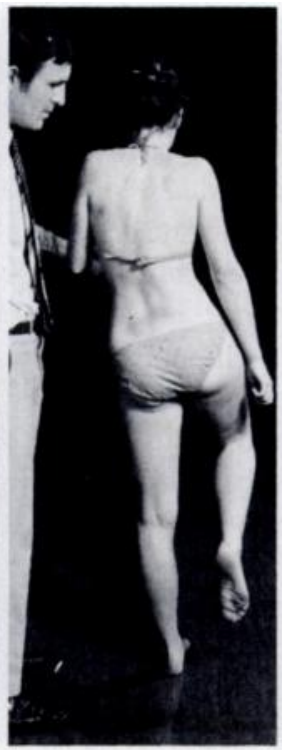

Fig. 10

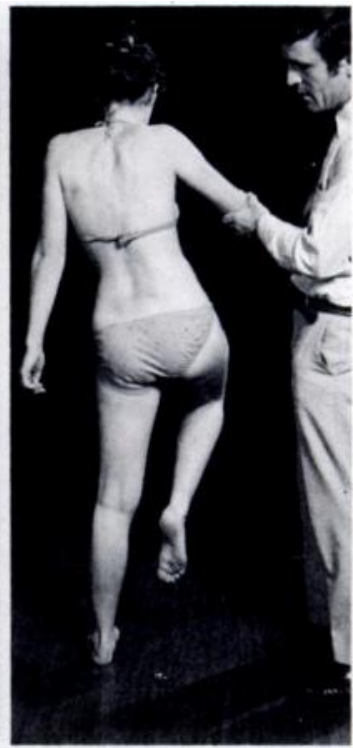

Fig. II

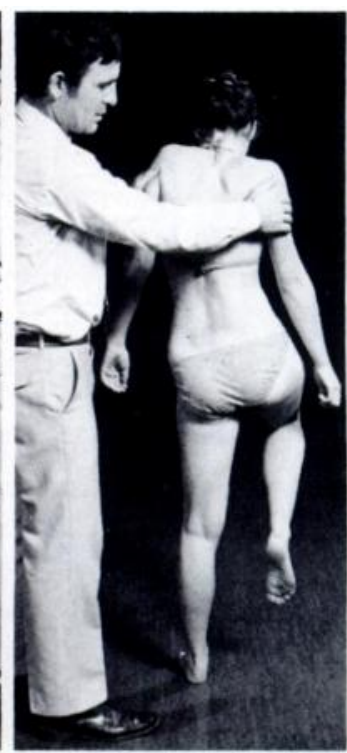

Fig. 12

The suggested method of performing the Trendelenburg test: Figure 7-The examiner stands behind the patient. Figure 8-The patient is asked to raise one leg off the ground with the hip flexed between 0 and 30 and to balance herself. Figure 9-The patient is asked to raise the non-stance side as high as possible. Figure 10-The examiner may support the patient by holding the arm on the other side. Figure $11-$ The examiner may not support the non-stance arm as this may act as a fulcrum for latissimus dorsi. quadratus lumborum, and the paraspinal muscles. Figure 12 --If there is significant trunk shift to the stance side this is corrected by gently aligning the trunk over the stance-side hip and watching the relation of the pelvis to the ground.

costal margin and the iliac crest when they attempted to elevate the pelvis.

\section{A STANDARD TRENDELENBURG TEST}

As a result of our observations, we have formulated a standard method of performing the Trendelenburg test; if this is used the response can be interpreted in a clinically meaningful way. To perform the test properly does take time, and its accurate assessment demands the full understanding and co-operation of the patient.

\section{How to do the test}

1. The examiner stands behind the patient and observes the angle between the pelvis (the line joining the iliac crests) and the ground (Fig. 7).

2. The patient is asked to raise from the ground the foot of the side not being tested, holding the hip joint at between neutral and 30 of flexion. The knee should be flexed enough to allow the foot to be clear of the ground in order to nullify the effect of the rectus femoris muscle. The position of the pelvis is again noted (Fig. 8). A supporting stick can be used in the hand only on the side of the weight-bearing hip; alternatively, both shoulders can be supported by the examiner so as to maintain balance without a stick (Fig. 12).

3. Once balanced, the patient is then asked to raise the non-stance side of the pelvis as high as possible (Fig. 9). The examiner may support the patient by holding the arm on the stance side (Fig. 10; compare with Fig. 11).

4. If the patient leans too far over to the side of the weight-bearing hip, the examiner corrects this by gentle pressure on the shoulders to bring the vertebra prominens approximately over the centre of the hip joint and the weight-bearing foot (Fig. 12).

\section{Interpretation}

(a) The response is NORMAL (i.e. the test is "negative") if the pelvis on the non-stance side can be elevated as high as hip abduction on the stance side will allow, and providing this posture can be maintained for 30 seconds with the vertebra prominens centred over the hip and foot.

(b) The response is $\mathrm{ABNORMAL}$ (i.e. the test is "positive") if this cannot be done. This includes responses where the pelvis is elevated on the non-stance side above the stance side, but where this elevation is not maximal.

(c) The response is also ABNORMAL if the pelvis can be lifted on command, but can not be maintained in that position for 30 seconds. The time taken before the pelvis starts to fall is recorded. By introducing a time element, the Trendelenburg test can be objectively recorded for comparison purposes. Obviously the response described in (b) constitutes a zero time Trendelenburg test.

Non-valid responses. In the presence of back or leg pain or of deformity, or if the patient is uncooperative because of age or mental status, inappropriate responses may arise (Table II). An abnormal response (positive test) in these circumstances can be misleading. However, if the test is negative that is significant--it means that the subject does not have abnormal hip mechanics. 


\section{Significance}

Neurological disorders. MRC Grade 5 abductor muscle strength was required to produce a normal response. All subjects whose hip abductor power was Grade 4 or less had abnormal responses at times between 0 and 25 seconds. Some patients in this group were able to elevate the pelvis, but not to the full extent. We considered this to be a positive Trendelenburg test at 0 seconds.

One subject who had Grade 5 strength on clinical testing had a delayed (or timed) positive Trendelenburg test at 15 seconds. Only one subject with Grade 3 hip abductors had an initial negative response which became positive at 5 seconds (a delayed positive test). He had a good rectus femoris muscle which probably helped. The two subjects with isolated nerve root entrapment of L5 or SI had negative responses.

Mechanical disorders. Congenital dislocation of the hip. The Trendelenburg test was always positive in subjects with congenital dislocation of the hip (Fig. 13). One patient who had been treated by bilateral replacement

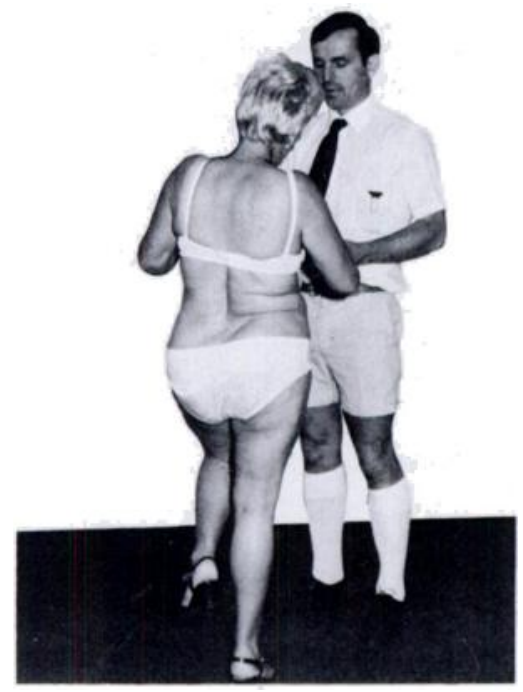

Fig. 13

A 48-year-old woman with congenital dislocation of the hip showing a positive Trendelenburg response despite supporting her body weight with her hands.

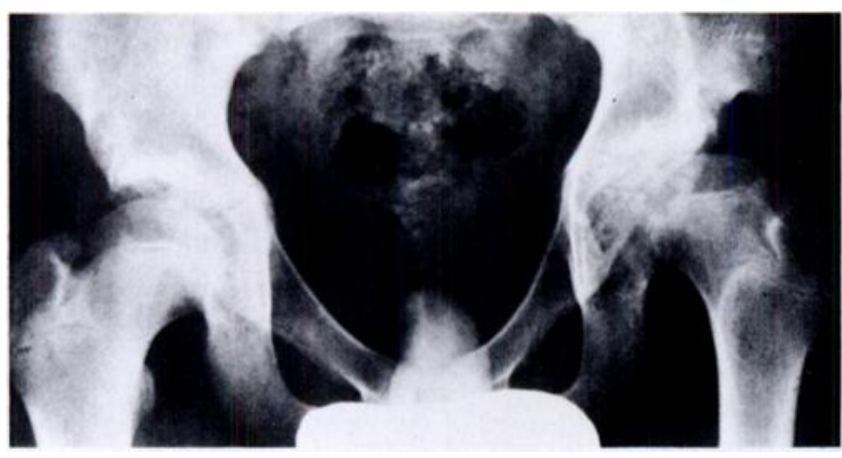

Fig. 14

Anteroposterior radiograph of a nine-year-old boy with a subluxating hip. His initial Trendelenburg response was negative but within 15 seconds he had a delayed positive test. arthroplasty had a normal response when examined three years after surgery.

Subluxating hips. Mitchell (1973) has described the importance of the delayed (timed) Trendelenburg test in assessing clinical deterioration in adolescents with acetabular dysplasia. Our two patients with subluxating hips both had positive (timed) Trendelenburg tests, one at 15 and one at 20 seconds (Fig. 14).

Coxa vara. The test may be positive or negative depending on the femoral neck angle and the presence of degenerative changes. With femoral neck angles of up to 100 the response can be normal. One child with an angle of 90 after a varus osteotomy had a positive test at 20 seconds.

Slipped femoral capital epiphysis. The Trendelenburg test was not altered by the rotation of the femoral head in relation to the femoral neck and the subjects examined all had negative responses if they were painfree.

Perthes' disease. The Trendelenburg test was not altered by the size of the femoral head. However, if there is incongruity or hinge abduction the test can become positive, and a delayed positive response was seen in one subject (Fig. 15).

Arthritis of the hip. Variable responses were observed but the type of response did not vary in the same individual when studied at different times. Obviously pain or progression of the disease would be expected to alter the response, particularly in respect to reduction of time of the normal response.

Leg length inequality after hip arthroplasty. Two patients with up to $2 \mathrm{~cm}$ of shortening above the intertrochanteric line after hip replacement had negative Trendelenburg tests (i.e. normal responses).

Avulsion of the greater trochanter after hip arthroplasty. In the absence of pain, the Trendelenburg test was particularly valuable some time after operation. Where the osteotomy gap was greater than $2 \mathrm{~cm}$ the Trendelenburg test was positive either immediately (zero time) or with a delayed positive response.

Fractured neck of femur. Patients who had unstable fixation with Ender or Zickel nails had a positive response

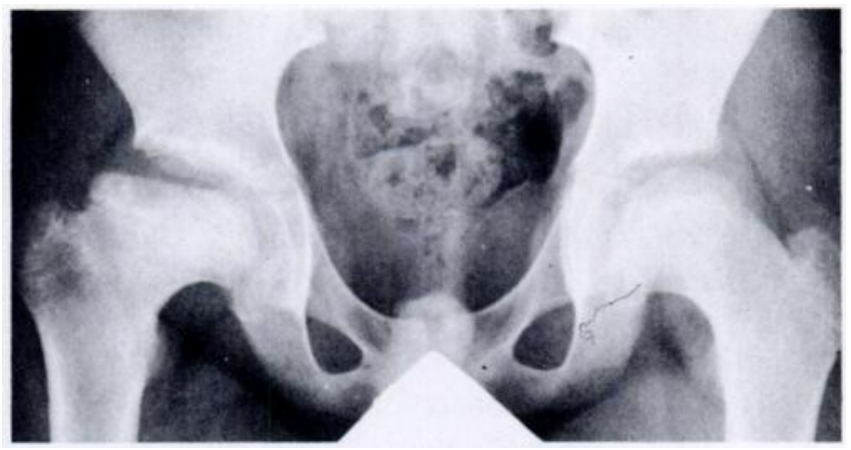

Fig. 15

Anteroposterior radiograph of a 10-year-old boy with coxa magna and hip joint incongruity as a sequel of Perthes' disease. He had a delayed positive test at 20 seconds. 
until the fracture was united radiologically. These people were tested initially 8 to 10 weeks after operation and had no pain at the time of their initial Trendelenburg response, which was positive. Two malunited fractures also had positive responses.

Avascular necrosis of the femoral head. Hip pain made proper assessment of these cases difficult. However, one of our four patients had only mild symptoms; his test was positive at 20 seconds on the first examination, and at 25 seconds on the next day. In the other patients pain prevented adequate assessment.

Spinal disorders. Stiffiness. Totally stiff spines, as in ankylosing spondylitis, did not affect the test unless there was also abnormality of the hip or gross spinal deformity.

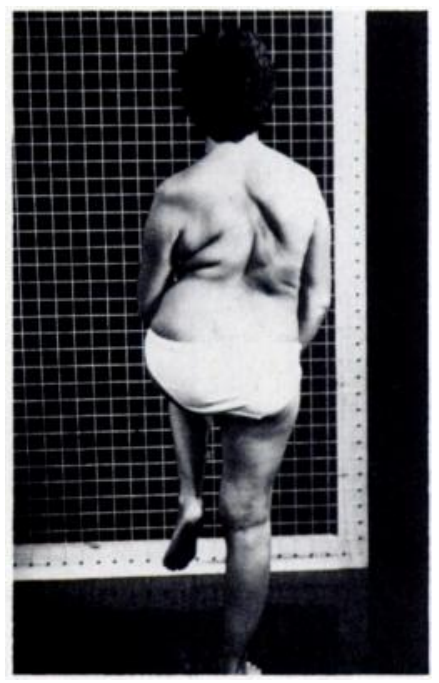

Fig. 16

This 43-year-old lady's hip abductors had normal strength. However, she was unable to raise her pelvis on the left above the horizontal because of impingement between the iliac crest and costal margin as the result of severe scoliosis.

Deformity. Kyphosis did not affect the outcome. Severe scoliosis, however, may lead to impingement between the lower costal margin and the iliac crest and give a falsepositive test (Fig. 16).

Pain. Nerve root irritation can lead to false-positive results, but back pain itself did not lead to abnormal responses.

\section{DISCUSSION}

When asked to stand on one leg, and follow a standard routine to ensure hip abductor muscle contraction on the same side, patients may respond to the standardised Trendelenburg test in one of three ways. Only one response is normal; the other two are abnormal. An ability to assume the normal response must be absolute, and if the pelvis drops on the non-stance side within 30 seconds the Trendelenburg test is positive. The use of a timer is an essential part of the Trendelenburg test, and, indeed, makes it an objective measure of severity of altered hip mechanics.

However, the presence of pain, poor balance and either lack of co-operation or understanding by the patient can lead to false-positive tests, because the test cannot be properly performed. The reason for falsenegative tests is that the subject uses muscles above the pelvis to elevate the non-weight-bearing side of the pelvis, or shifts the torso well over the weight-bearing side; these can be called "trick movements". Variable responses were noted in some patients less than seven years of age, and the test is of no value in children under four. Nevertheless, if the Trendelenburg test is carefully performed, it is an accurate clinical sign with prognostic implications.

Inman (1947) measured the torque strength about the hip with the pelvis in different postures with respect to the ground. Our electromyographic results confirm his findings that little abductor muscle strength/activity is necessary to maintain a balanced posture with the pelvis dropped (as in Response 3) on the non-weight-bearing side. As the pelvis rises on this side there is increase of abductor muscle activity provided that the torso is centred over the hip.

Functional assessment of a joint is important in the clinical assessment of patients. Observation of gait is probably performed less often than is desirable because of limitation of space. The Trendelenburg test allows for functional assessment in a confined space, and is a more valuable clinical sign than many static tests. It can also be easily recorded on film or videotape.

It is our belief that a patient who has an abnormal response to the Trendelenburg test as described in this paper has an inefficient gait, and therefore becomes easily fatigued. With a little practice, the test is not difficult to perform and interpret. Timing is an essential part of the test; it provides an objective measure of improvement or deterioration in the neuromuscular or mechanical function of the hip.

Trendelenburg's original observations were precise and clear, and his interpretations accurate. We support the need for meticulous clinical examination in order to provide correct diagnosis and we recommend the use of the standardised timed Trendelenburg test in the assessment of function and malfunction of the hip.

\section{Conclusions}

1. The Trendelenburg test is a useful part of clinical examination if performed and interpreted correctly. We have described a standard method for performing the test.

2. False-positive and false-negative responses may occur, but their interpretation can be clarified if the test is properly performed.

3. The use of a timer when performing the test is essential, and allows measurement of a "delayed abnormal" response.

\section{REFERENCES}

Inman VI. Functional aspects of the abductor muscles of the hip. $J$ Bone Joint Surg [Br] 1947;29:607-19.

Mitchell GP. The delayed Trendelenburg hip test. Int Congr Ser 1973; 291: 1113.

Rang M. Anthology of orthopaedics. Edinburgh: E\&S Livingstone, $1966 ; 139-43$. 